\title{
Evaluación del etiquetado de quesos de origen animal y "veganos"
}

\author{
Assessment of the labelling of cheese of animal origin and vegan \\ Dulce I. Hernández-Lazcano ${ }^{a}$, Vianey López-Ordoñez, ${ }^{b}$, José G. Pérez-Brandt ${ }^{c}$, Esther \\ Ramírez-Moreno ${ }^{d}$
}

\begin{abstract}
:
Cheese is the product made from milk curd, which can be fresh, ripe or processed. These products must meet the criteria of the established standards. Actually, alternatives such as "vegan" cheeses can be found on the market. The aim of this work was to evaluate the nutritional labelling of the different types of dairy cheeses and "vegan cheeses" sold on the market. Cheeses are protein foods being between 5 and $25 \mathrm{~g} / 100 \mathrm{~g}$ depending if the cheese is fresh or ripe, while the vegan cheeses had a very low content of this nutrient. Fat content was similar between the cheeses studied and the differences with the vegan cheeses was the presence of the use of coconut oil. There was no difference in sodium and fat content between dairy cheese and vegan products, and not all brands showed the calcium content in the label. The use of different additives where the use of rubber in the cheese industry is predominant. Finally, the price variability was very wide, being more expensive" vegan" cheeses.

Cheese is a protein product that can be part of the diet to meet the requirement of this nutrient, however, in recent years has become fashionable the consumption of "vegan" cheeses justifying that they are healthier, these, unlike cheeses of animal origin, are made with gums, oils of vegetable origin and have a low protein content, in addition to the fact that the sodium intake is high and similar to that of a cheese made with milk. Therefore, moderate consumption of both products should be considered, as it could have adverse health effects.
\end{abstract}

Keywords:

Cheese, vegan cheese, nutrition labelling

\section{Resumen:}

El queso es el producto elaborado con la cuajada de la leche, los cuales pueden ser frescos, madurados ó procesados. Estos productos deben cumplir con los criterios de las normas establecidas. Actualmente, en el mercado se pueden encontrar alternativas, tales como los quesos "veganos". El propósito del presente trabajo fue evaluar el etiquetado nutrimental de los diferentes tipos de quesos de origen lácteo y "quesos veganos" expendidos en el mercado. Los quesos son alimentos proteicos encontrándose entre 5 y $25 \mathrm{~g} / 100 \mathrm{~g}$ dependiendo si el queso es fresco o madurado, mientras que los quesos veganos presentaron un contenido muy bajo de este nutrimento. El contenido de grasa fue similar entre los quesos estudiados y las diferencias con los quesos veganos fue la presencia del uso de aceite de coco. No hubo diferencia en el contenido de sodio y grasas entre los quesos lácteos y los productos veganos, además, no todas las marcas presentaron el contenido de calcio en el etiquetado. El uso de diferentes aditivos en donde es predominante el uso de gomas en la industria quesera. Por último, la variabilidad de precios fue muy amplia, siendo más caros los quesos "veganos".

El queso es un producto proteico que puede formar parte de la dieta para alcanzar los requerimientos de este nutriente, sin embargo, en los últimos años se ha puesto de moda el consumo de quesos "veganos" justificando que son más saludables, estos, a diferencia de los quesos de origen animal, son elaborados con gomas, aceites de origen vegetal y tienen un bajo aporte proteico, además de que el aporte de sodio es alto y similar al de un queso elaborado con leche. Por lo que se debe considerar el consumo moderado de ambos productos, ya que pudiera tener efectos adversos a la salud.

Palabras Clave:

Queso, quesos veganos, etiquetado nutrimental

\footnotetext{
a Autor de Correspondencia, Universidad Autónoma del Estado de Hidalgo, https://orcid.org/0000-0001-9517-8351, Email: dulce96isui@gmail.com

b Universidad Autónoma del Estado de Hidalgo, Instituto de Ciencias de la Salud, https://orcid.org/0000-0003-3701-9314, Email: vianey.or04@gmail.com

c Universidad Autónoma del Estado de Hidalgo, Instituto de Ciencias de la Salud, https://orcid.org/0000-0001-6246-1847, Email: jose.guadalupe.perez.brandt@gmail.com

Universidad Autónoma del Estado de Hidalgo, Instituto de Ciencias de la Salud, https://orcid.org/0000-0002-9928-8600, Email: esther_ramirez@uaeh.edu.mx
} 


\section{Introducción}

El queso, por definición, es el producto elaborado con la cuajada de leche estandarizada y pasteurizada de vaca o de otras especies animales. Estos productos se clasifican en: quesos frescos, con un alto contenido de humedad, sabor suave y no tienen corteza; quesos madurados, de pasta dura, semidura o blanda, con o sin corteza y se someten a un proceso de maduración con microorganismos bajo condiciones controladas (tiempo, temperatura y humedad); quesos procesados, caracterizados por ser mezclas de quesos, fusión y emulsión con sales fundentes, algunos aditivos e ingredientes opcionales.' La NOM-223-2018 establece que el queso debe de contener como mínimo $10 \%$ de proteína y una humedad máximo del $80 \%$. Actualmente en el mercado se ha incrementado la venta de quesos veganos, que tienen como objetivo presentar una alternativa de productos para la población que no consume leche o sus derivados.

\section{Objetivo}

Evaluar la composición nutrimental y aditivos presentados en el etiquetado de diferentes tipos de quesos y quesos veganos expendidos en supermercados de la ciudad de Pachuca, Hgo.

\section{Metodología}

Se evaluaron seis tipos de queso de diferentes marcas de origen animal $\mathrm{y}$ de origen vegetal, estos últimos conocidos comercialmente como "veganos", en diferentes supermercados de la ciudad de Pachuca, Hgo. Posteriormente se revisó el etiquetado nutrimental de estos productos y la información (contenido energético, proteínas, grasas, carbohidratos, azúcares, sodio, calcio, aditivos y precio). La información fue compilada en una base de datos. Los datos recabados de la composición nutrimental fueron calculados en base a $100 \mathrm{~g}$ para poder establecer comparaciones entre los productos evaluados, aunque las unidades comerciales variaron entre los $140 \mathrm{~g}$ y 500 g.

Se utilizaron las NOM-121, NOM-223 y NOM-243 para verificar el cumplimiento del contenido nutrimental.

\section{Resultados y Discusión}

En la tabla 1 se muestran los 6 tipos de quesos de 8 marcas evaluadas de origen animal y 3 marcas "veganas". En estos productos se resalta principalmente su contenido de proteínas, grasas y sodio.
Tabla 1. Contenido nutrimental de diferentes tipos de quesos de

\begin{tabular}{|c|c|c|c|c|c|c|c|c|c|}
\hline origen & anim & & & $y$ & & egeta & & & $g / 100$ \\
\hline$\stackrel{ }{2}$ & $\frac{\mathbb{\pi}}{2}$ & 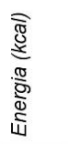 & 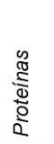 & $\begin{array}{l}\mathscr{J} \\
\mathbb{8} \\
\mathbb{0} \\
\text { (1) }\end{array}$ & 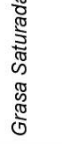 & $\begin{array}{l}\frac{2}{0} \\
\frac{0}{00} \\
\frac{0}{0} \\
\frac{0}{00} \\
0\end{array}$ & 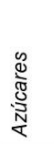 & 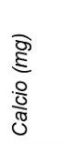 & $\begin{array}{l}\text { के } \\
\text { है } \\
\text { के } \\
\text { ल }\end{array}$ \\
\hline & & & Orige & Anim & & & & & \\
\hline Americano & Esmeralda & 249.1 & 10.2 & 18.2 & 12.5 & 10.8 & 0.57 & NM & 800 \\
\hline & FUD & 277.7 & 12.2 & 20 & 12.7 & 12.2 & 7.7 & NM & 1400 \\
\hline & La Villita & 308.5 & 6.2 & 25.1 & 13.1 & 14.2 & 5.7 & NM & 1000 \\
\hline & Lala & 300 & 15.5 & 23.8 & 15.5 & 6.1 & 3.8 & 394.4 & 1650 \\
\hline & Philadephia & 200 & 13 & 11 & 7 & 11.6 & 11 & NM & 1326 \\
\hline Cheddar & Navarro & 356.6 & 25.6 & 25.3 & 15 & 6.3 & 0 & NM & 553.3 \\
\hline Cottage & Lala & 93.3 & 10 & & 3 & 2.3 & 2.3 & 103.3 & 400 \\
\hline & Lyncott & 101 & 12 & 4.2 & 3.1 & 3.7 & 2.8 & NM & 300 \\
\hline Gouda & $\begin{array}{l}\text { Noche } \\
\text { Buena }\end{array}$ & 380 & 24 & 31.2 & 19.2 & 1.2 & 1.2 & NM & 748 \\
\hline & Kroon & 366.6 & 23.3 & 30 & 20 & 0 & 0 & NM & 766.6 \\
\hline Mozzarella & FUD & 307.1 & 22.1 & 22.8 & 14.2 & 3.5 & 1.4 & NM & 921.4 \\
\hline Queso & Esmeralda & 249.1 & 10.2 & 18.2 & 12.5 & 10.8 & 0.57 & NM & 800 \\
\hline crema & Lala & 310 & 5 & 30 & 20 & 4.6 & 4.6 & 190 & 350 \\
\hline & Philadephia & 243.3 & 6 & 22 & 15.6 & 5 & 5 & NM & 503.3 \\
\hline & Great Value & 346.6 & 6.6 & 33.3 & 23.3 & 37.3 & 5 & NM & 500 \\
\hline & & & & anos & & & & & \\
\hline Americano & Daiya & 272.7 & 0 & 22.7 & 20.45 & 18.18 & 0 & 622.7 & 863.6 \\
\hline Cheddar & Daiya & 272.7 & 0 & 22.7 & 20.45 & 18.18 & 0 & 618.1 & 818.1 \\
\hline Cottage & $\begin{array}{l}\text { Veggie } \\
\text { Delicatessen }\end{array}$ & 220 & 6.6 & 20 & 16.6 & 3.3 & 0 & NM & 376.6 \\
\hline Gouda & Daiya & 272.7 & 0 & 22.7 & 20.4 & 18.18 & 0 & 618.1 & 863.6 \\
\hline Mozzarella & $\begin{array}{l}\text { Veggie } \\
\text { Delicatessen }\end{array}$ & 256.6 & $<1$ & 20 & 16.6 & 16.6 & 0 & NM & 633.3 \\
\hline $\begin{array}{l}\text { Queso } \\
\text { crema }\end{array}$ & Tofutti & 200 & 3.3 & 16.6 & 6.6 & 6.6 & 0 & NM & 400 \\
\hline
\end{tabular}

El contenido energético de los quesos depende del aporte de grasas y proteínas de estos productos. El queso cheddar presentó un mayor aporte energético ( $437 \mathrm{kcal}$ ) en comparación con el queso cottage $(93.3 \mathrm{kcal})$. Debido a que el queso cheddar es un producto que se obtiene después de un tiempo de maduración como muchos otros quesos, el contenido de humedad disminuye y el de proteínas y grasas se incrementa. A diferencia de quesos frescos como queso cottage o queso panela que inmediatamente después de su obtención es empacado manteniendo al producto con un alto contenido de humedad, con la composición muy similar a la leche de la cual proviene.

La NOM-223 establece que el contenido de proteínas mínimo de los quesos debe de ser de $10 \%$. El queso cottage tuvo el mínimo contenido de proteína de acuerdo a esta norma en comparación con los otros quesos como el queso cheddar (26.6 $\mathrm{g} / 100 \mathrm{~g}$ ). En cuanto a los quesos que no cumplen con el mínimo contenido de proteína están los quesos tipo crema y el queso americano de la marca "La Villita" que presentaron valores por debajo del $10 \%$. Por lo tanto, se recomienda buscar productos que tengan al menos $10 \%$ de proteína y la mínima cantidad de grasa.

El aporte de proteínas es mucho menor o nulo en los quesos "veganos" en comparación con los de origen animal, esto debido a que el ingrediente principal de los quesos de origen animal es leche. Las caseínas y proteínas lactoséricas, son de alto valor biológico y permiten una mejor retención de humedad y de los otros componentes del queso como la grasa contribuyendo con la textura sólida del queso. En el caso de los quesos veganos no son elaborados con proteína, su contenido es bajo $(<0.1 \mathrm{~g} / 100 \mathrm{~g})$, por lo que, la industria implementa el uso de gomas para darle la textura deseada a los quesos "veganos". ${ }^{5-6]}$

En relación con las grasas estas fluctuaron entre 4.2 a 36.6 g/100 $\mathrm{g}$ de producto Es importante destacar que la cantidad de grasas en los productos de origen animal en promedio es de 22.15 g/100 g, muy similar a los productos de origen vegetal (20.78 
g/100 g). La diferencia radica únicamente en el tipo de grasa en los productos, mientras que la grasa de los quesos es de origen lácteo, mientras en los quesos veganos es proveniente del aceite de coco.

Como se observa en la Tabla 1, no todos los productos informaron en sus etiquetas el contenido de calcio, sin embargo, de acuerdo con la NOM-243-2010, el contenido de este nutriente dependerá de las buenas prácticas de fabricación. La NOM-121-1994 indica que estos productos deben de contener por lo menos $0.3 \%$ (ó $300 \mathrm{mg}$ de calcio / $100 \mathrm{~g}$ de producto). Por lo que, los quesos evaluados cumplen con la norma, destacando que el queso Americano de la marca Daiya presentó el mayor contenido en calcio $(622.7 \mathrm{mg} / 100 \mathrm{~g})$, en comparación con los demás.

Esta misma norma, establece que los quesos deben tener como máximo $0.3 \%$ (ó $300 \mathrm{mg}$ de sodio / $100 \mathrm{~g}$ de producto). Con lo anterior, se puede observar que los quesos presentes en la tabla 1 sobrepasan el valor establecido por la norma. El queso con menor contenido de sodio y que cumple con la norma, en comparación con los demás, es el queso cottage de la marca Lyncott (300 mg/ $100 \mathrm{~g}$ de producto), los demás estuvieron por encima de esta cantidad. Por tanto, de acuerdo con las recomendaciones de la OMS ( 2 g/día), 100 g de producto estaría aportando hasta el $15 \%$ de la ingesta diaria recomendada (IDR) para la población.

Tabla 2. Ingredientes y aditivos de quesos de origen animal y veganos

\begin{tabular}{|c|c|c|c|c|c|c|}
\hline Tipo & Leche & Cuajo & Sal & $\begin{array}{c}\text { Aceite } \\
\text { de coco }\end{array}$ & $\begin{array}{c}\text { Cloruro } \\
\text { de } \\
\text { calcio }\end{array}$ & Gomas \\
\hline \multicolumn{7}{|c|}{ Origen Animal } \\
\hline Mozzarella & $x$ & $x$ & $x$ & & & $x$ \\
\hline Cheddar & $x$ & $x$ & $\mathrm{X}$ & & & $x$ \\
\hline Americano & $\mathrm{x}$ & $x$ & $x$ & & $x$ & $x$ \\
\hline $\begin{array}{l}\text { Queso } \\
\text { crema }\end{array}$ & $\mathrm{x}$ & $x$ & $\mathrm{X}$ & & & $x$ \\
\hline Cottage & $x$ & $x$ & $\mathrm{X}$ & & & $x$ \\
\hline \multicolumn{7}{|c|}{ Veganos } \\
\hline Mozzarella & & & $X$ & $\mathrm{X}$ & $\mathrm{X}$ & $X$ \\
\hline Cheddar & & & $x$ & $x$ & & $x$ \\
\hline Gouda & & & $\mathrm{X}$ & $x$ & $x$ & $x$ \\
\hline Americano & & & $\mathrm{x}$ & $x$ & & $x$ \\
\hline $\begin{array}{l}\text { Queso } \\
\text { crema }\end{array}$ & & & $x$ & $x$ & & $x$ \\
\hline Cottage & & & $\mathrm{x}$ & $x$ & $x$ & $x$ \\
\hline
\end{tabular}

Como se muestra en la Tabla 2 podemos ver los principales ingredientes de los quesos industrializados tanto de origen animal como vegetal. Cabe destacar que la adición de aditivos en los quesos ayudará tanto en la disminución de humedad o bien, a que estos no ganen mayor humedad durante su almacenamiento, por otro lado, ayudarán en la caracterización de firmeza. Además de la sal, un ingrediente clave son las gomas, las cuales proporcionan características a los quesos. De acuerdo a la NOM-243-2010 y el Codex Stan 283-1978 indican que el contenido de gomas en los quesos se adiciona según las buenas prácticas de fabricación que se tengan en la industria para mejorar mejores características como una textura más suave. ${ }^{6}$ Por otra parte, se puede observar en la Tabla 3 , que existe una gran variabilidad entre los precios de quesos de origen animal y los de origen vegetal. Los quesos de origen animal resultaron ser más económicos, con precios que fluctúan entre los $\$ 10$ a 20.18 por cada $100 \mathrm{~g}$ de producto, dependiendo el tipo de queso y marca. Por el contrario, los precios de quesos "veganos" van de $\$ 20$ a 58 pesos por cada 100 g de producto, por lo que da como resultado un producto poco accesible económicamente y con un contenido pobre de proteínas, ocasionando un producto con una baja relación en cuanto a precio/calidad.

Tabla 3. Precios de quesos de origen animal y vegetal.

\begin{tabular}{llc} 
Tipo & Marca & Precio por $\mathbf{1 0 0} \mathbf{g}(\mathbf{M N X})$ \\
\hline Americano & \multicolumn{1}{c}{ Origen Animal } & \\
& Esmeralda & 10 \\
& FUD & 15.06 \\
& La Villita & 12.57 \\
Cheddar & Lala & 16.67 \\
Cottage & Philadephia & 15.63 \\
& Navarro & 17.88 \\
Gouda & Lala & 11.23 \\
& Lyncott & 9.87 \\
Mozzarella & Noche Buena & 20.18 \\
Queso crema & Kroon & 18.13 \\
& FUD & 18.00 \\
& Esmeralda & 14.29 \\
& Lala & 13.16 \\
& Philadephia & 17.93 \\
\hline Americano & Great Value & 11.58 \\
Cheddar & & \\
Cottage & Vaiya & 58.64 \\
Gouda & Daiya & 58.64 \\
Mozzarella & Veggie Delicatessen & 20.45 \\
Queso crema & Daiya & 58.64 \\
\hline
\end{tabular}

\section{Conclusión}

Los quesos son un producto proteico que incorporado a la dieta permite cubrir los requerimientos diarios de este nutrimento. Cabe mencionar, que en los últimos años se ha puesto de moda el consumo de quesos "veganos", con la justificación de que son más saludables y buenos para la salud o bien, por el simple hecho de ser más conscientes de la explotación de los animales. Sin embargo, a diferencia del queso elaborado con leche, los productos veganos son productos elaborados con gomas y con un bajo aporte proteico y aceites de origen vegetal, por lo cual se debe considerar ampliamente su consumo.

En general ambos productos aportan una gran cantidad de sodio por porción consumida tanto en los quesos "veganos" y de origen animal sobre todo madurados, por lo que se debe considerar un consumo moderado de estos productos, ya que pudiera tener efectos adversos en la salud.

\section{Referencias}

[1]

NOM-243-SSA1.

Dirección: http://dof.gob.mx/nota detalle.php?codigo $=5160755 \&$ fecha $=27 / 09 / 2010$ Actualización: 27/09/2010; Acceso: 9/03/2020.

[2] NOM-223-SCFI-/SAGARPA. Dirección: https://dof.gob.mx/nota detalle.php? codigo $=5549319 \&$ fecha=31/01/2019 Actualización: 31/01/2019; Acceso: 16/03/2020.

[3] NOM-121-SSA1. Dirección: http://www.salud.gob.mx/unidades/cdi/nom/121ssa14.html Actualización: 1994; Acceso: 16/03/2020

[4] OMS. Dirección: https://apps.who.int/iris/bitstream/handle/10665/85224/WHO_NMH_NH D 13.2 spa.pdf;jsessionid=F83BDFB7686BC84F3F2D4589907BACA4? sequence $=1$ Actualización: 2013. Acceso: 16/03/2020.[5] Rojas, D, Figueras, F, Durán, S. (2017). Ventajas y desventajas nutricionales de ser vegano o vegetariano. Rev. Chil. Nutr. 44(3)

[6] OEA. Dirección: http://portal.oas.org/LinkClick.aspx?fileticket=O51xfikk6CU\%3D\&ta Actualización: 2000; Acceso: 18/03/2020

[7] Ochoa-Flores, Hernández-Becerra, J, López-Hernández, E. y GarcíaGalindo, H., 2013. Rendimiento, Firmeza y Aceptación Sensorial de Queso Panela Adicionado con Estabilizantes. Universidad y Ciencia. 29(3): 277286. 